\title{
The Rossiter-McLaughlin Effect for Planets and Low-Mass Binaries
}

\author{
Amaury H. M. J. Triaud \\ Observatoire Astronomique de l'Université de Genève, Chemin des Maillettes, 51, CH-1290 \\ Sauverny, Switzerland \\ email: Amaury.Triaud@unige.ch
}

\begin{abstract}
The Rossiter-McLaughlin effect occurs during the eclipse or transit of an object in front of another one. In our case, it appears as an anomaly on the radial velocity Doppler reflex motion. The modelling of that effect allows one to measure the sky-projected angle between the rotation spin of the primary and the orbital spin of the secondary. In the case of exoplanets, it gave clues about the formation of the hot Jupiters. In this paper, I will talk about how the data are acquired, how models are adjusted to them, and which results have been made.
\end{abstract}

Keywords. planetary systems, binaries: eclipsing, binaries: spectroscopic, methods: data analysis.

\section{Introduction}

The discovery of the first hot Jupiter, 51 Peg b, by Mayor \& Queloz (1995) was a surprise. Gas giants such as this planet were supposed to form beyond the so-called snow-line, where ices and dust can coagulate to form cores large enough to attract a large amount of gas onto them (e.g. Pollack et al. 1996 and Alibert et al. 2005). Orbital migration had to happen.

Two main pathways were quickly proposed. One required planet-disc interactions which apply a torque on the planet leading to inward migration. The ideas have been put forward in Goldreich \& Tremaine (1980), Lin et al. (1996) and Ward (1997). Since it was first proposed, the study of disc migration has progressed a lot. One of its main predictions is that planets stay in the plane of the protoplanetary disc, which is aligned with the equatorial plane of its star. The second pathway relied on multi-body gravitational interactions as outlined in Rasio \& Ford (1996) for the case of planet-planet interactions. The main point is that a planet is placed via those interactions on an inclined, highly eccentric orbit. At periastron passage, tidal dissipation leads to angular momentum loss which shrinks the semimajor axis and circularises the planet's orbit (see Fabrycky \& Tremaine 2007 or Nagasawa et al. 2008).

In order to estimate the stellar rotation, Holt (1893) proposed that a colour effect should be visible when two stars eclipse each other. This colour effect was measured as a radial velocity anomaly by Rossiter (1924) and McLaughlin (1924) (see also Simon Albrecht's contribution in these proceedings). This effect was modelled notably by Kopal (1942) and Hosokawa (1953) using the $\alpha$ functions.

In the context of extrasolar planets, the Rossiter-McLaughlin effect was first observed by Queloz et al. (2000). Since then, a fast growing number of measurements have been collected which are refining our ideas about the formation of hot Jupiters. In order to interpret the data correctly, one has to build a statistical picture. This requires a large number of subjects. Hot Jupiters are rare planets: they occur around about one star in 200 (eg. Naef et al. 2005 and Howard et al. 2011). Fortunately, wide angle ground-based 



Figure 1. The joint probability distribution, output of an MCMC interpolating within the Geneva stellar evolution tracks in a modified H-R diagram using $\rho_{\star}^{-1 / 3}$ against $T_{\text {eff }}$. The aim is use the property that we measure $\rho_{\star}^{-1 / 3}$ from a planetary transit to estimate the planet's host mass and age. 1, 2 and $3 \sigma$ confidence intervals are overplotted. On the left we see the result on mass tracks of constant metallicity, on the right we see tracks for a constant mass at different metallicities. Thus our host star, here WASP-8, is about $0.99 \pm 0.05 M_{\odot}$.

transit surveys have now found more than 100 such objects. One of those is the WASP survey (Pollacco et al. 2006). The aim of the WASP survey is to find transiting planets on stars bright enough so one could study them more in detail. The survey aimed at stars between $\mathrm{V}=9$ and $\mathrm{V}=13$. Across the years, we have found close to 60 planets. In addition, in our search for transiting -eclipsing- brown dwarfs, we have detected a large number of low-mass eclipsing binaries consisting mostly of $\mathrm{F}+\mathrm{M}$ and $\mathrm{G}+\mathrm{M}$ pairs.

\section{Characterising planets}

The photometric transit is modelled using Mandel \& Agol (2002) or Giménez (2006a). The Rossiter-McLaughlin effect is adjusted using the formalism of Giménez (2006b) based on Kopal's $\alpha$ functions. The adjustment of those models to the data is done using a Markov Chain Monte Carlo algorithm (MCMC). This is a powerful method which allows us to solve for highly non-linear models, on datasets combining multi-band, multiinstrument, multi-epoch photometry and spectroscopy. The interest of such a combined analysis is to produce the best possible compromise between all datasets, which describe the same physical phenomenon, observed differently. For instance, long time-base photometry will very precisely determine the period and phase of the object we will study. When adjusting the radial velocity, there really is only one parameter which is effectively variable: the semi-amplitude. This allows us to refine our error bars on that parameter and, consequently, on the mass of our planet. Similarly, the photometric transit will determine the ratio of radii and impact parameter, so when a model is adjusted for the Rossiter-McLaughlin effect, the fit only varies two quantities: the $V \sin I$ and the spin/orbit angle $\beta$. This allows us to detect lower signal-to-noise since we have fewer free parameters. By combining all information, we make sure that all parameters are consistent with each other and with the entire dataset.

Another interest of the MCMC is that it produces probability density distributions marginalised for each parameter instead of $\chi^{2}$ maps, which makes it very easy to produce 


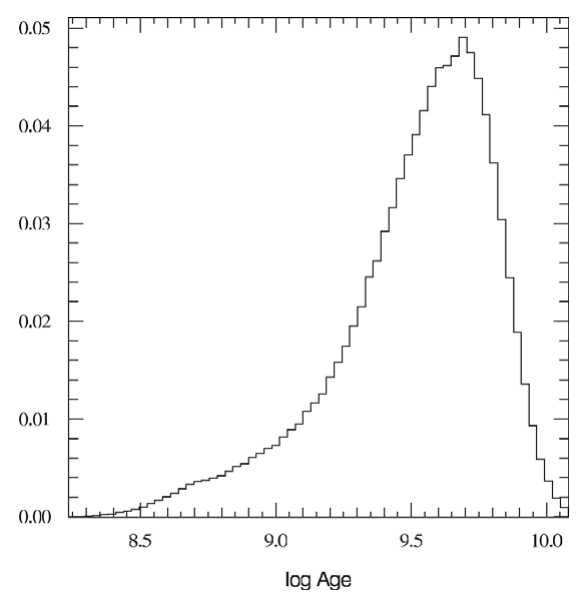

Figure 2. Marginalised probability density function for stellar age, estimated from the distribution over the stellar evolution tracks presented in Figure 1. WASP-8 is $5 \pm 2.5$ Gyr old.

robust confidence intervals. The concept of the MCMC is well-described in Gregory (2005). They have been in use by the WASP team and are presented in papers such as Cameron et al. (2007), Gillon et al. (2009), and Triaud et al. 2009.

Sozzetti et al. (2007) remarked that a planetary photometric transit measures directly the mean density of the host star, $\rho_{\star}$. This parameter can be used instead of the more traditional $\log g$ to interpolate within stellar evolution tracks in order to get an estimate of the stellar mass $M_{\star}$. To do that, one also needs the $T_{\text {eff }}$ and the metallicity $Z$. This process has now been included in the MCMC in the same spirit as before: that of getting the best compromise out of the data. For each step that the MCMC proposes, the Geneva stellar evolution tracks (Mowlavi et al. 2012) are interpolated in $\left(\rho_{\star}^{-1 / 3}, Z, T_{\text {eff }}\right)$ space in order to determine $M_{\star}$ and the stellar age (Triaud 2011). We thus end up with probability density functions for those two values. Errors in the models can also be accounted for. An example is given in Figure 1 and an age estimate given in Figure 2. We see that the tracks are evolving almost parallel to the value of $\rho_{\star}^{-1 / 3}$. This means that for a given $T_{\text {eff }}$ and $Z$, the stellar age is almost solely determined by $\rho_{\star}^{-1 / 3}$. In the case presented in the figures, the fit included only one high-precision photometric transit. Let's note here that GAIA, through distance measurements, will produce independent stellar radii, which added as priors in the above analysis should improve our accuracy and precision on stellar masses and ages.

\section{The spin/orbit angle survey}

Having detected a large number of planets, the WASP consortium endeavoured to learn the most possible about them. Part of that effort was to attempt to measure systematically the Rossiter-McLaughlin effect for each of our planets mostly using the HARPS spectrograph on the $3.6 \mathrm{~m}$ at La Silla, Chile. This led to a series of papers which showed that hot Jupiters are located on a large variety of angles, including some on retrograde orbits, instead of being coplanar as previously assumed. It also showed that tidal circularisation timescales are shorter than the realignment timescale as planets on circular, retrograde orbits were found. Some of the measurements and an interpretation of those results are presented in Triaud et al. 2010. Two effects are also shown in Figure 3. 

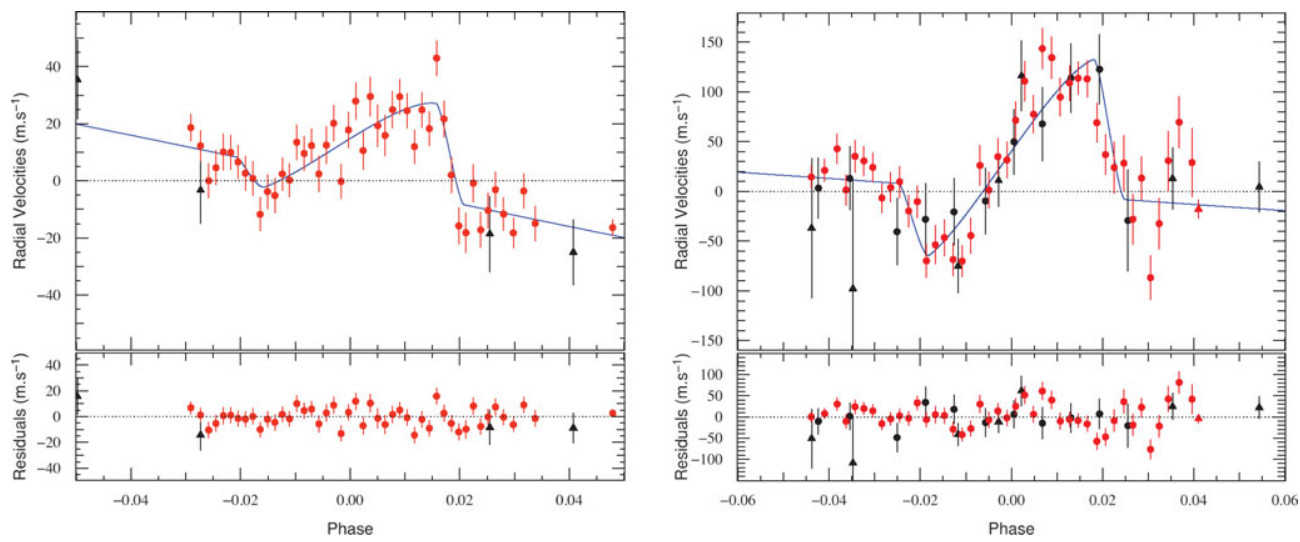

Figure 3. The R-M effect for WASP-15b and WASP-17b and residuals as appearing in Triaud et al. 2010. Circles are HARPS observations, triangles are CORALIE observations.

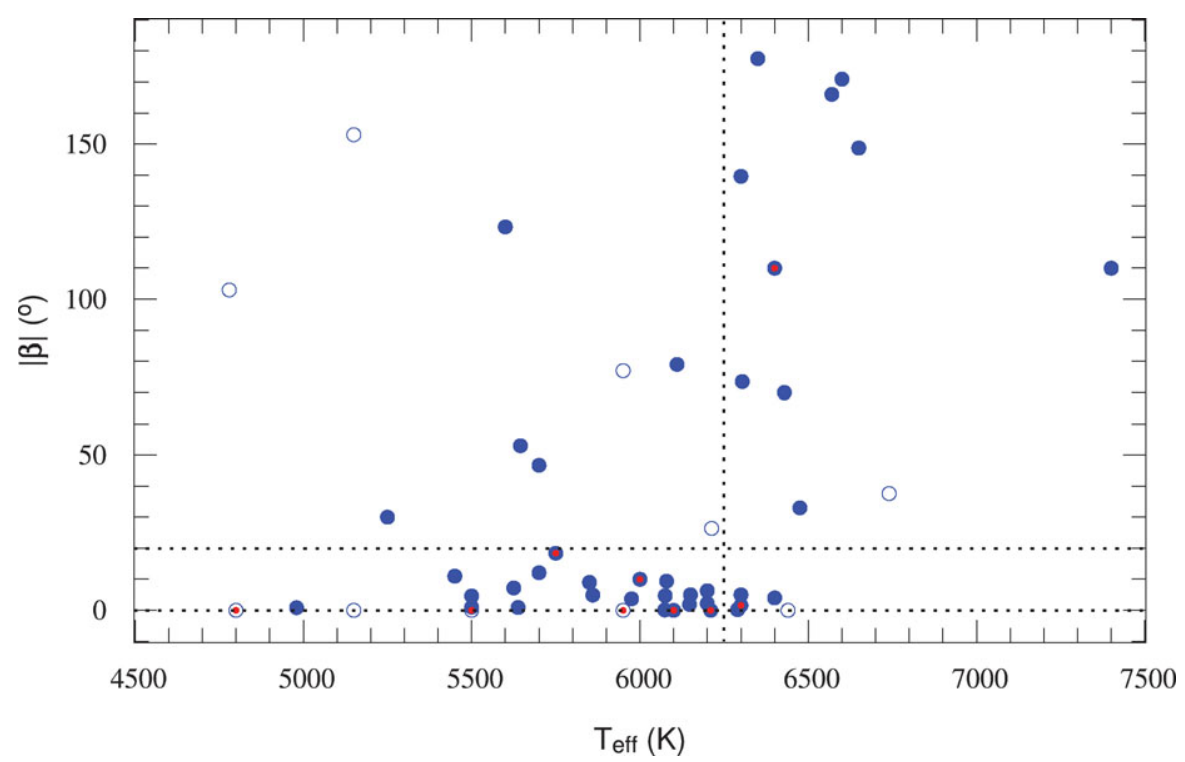

Figure 4. Sky-projected spin/orbit angle $\beta$ against $T_{\text {eff }}$. This plot is an update from that presented in Winn et al. (2010). Filled symbols show secured measurements. Open symbols are numbers likely to change dramatically due to low signal-to-noise detections or other issues. Little dots within symbols show as of yet unpublished measurements.

Winn et al. (2010) showed that when the spin/orbit angle $\beta$ (called $\lambda$ in their paper) is plotted agains the star's $T_{\text {eff }}$, a structure appears in the data. Aligned planets around hot stars are rare. Since that paper was published, a number of measurements have been made. An update of that plot is presented in Figure 4. With almost twice more data than when originally proposed, the pattern is essentially confirmed.

In addition to the planets that were discovered with WASP, we started characterising the orbits of single line $\mathrm{F}+\mathrm{M}$ and $\mathrm{G}+\mathrm{M}$ eclipsing binaries. We have now close to 50 wellsampled orbits. On 15 of those, we have also measured the Rossiter-McLaughlin effect using the CORALIE spectrograph, mounted on the Swiss $1.2 \mathrm{~m}$ Euler telescope at La 
Silla. The same tools used for analysing planets have been used, since those eclipsing M-dwarfs have sizes very similar to planets $\left(<2 R_{\text {Jup }}\right)$ while remaining very faint compared to their primary. So far, all spin/orbit angles have been found aligned in a marked difference with the planet population. According to Fabrycky \& Tremaine (2007) (and references there-in), high mass ratio binaries such as our $\mathrm{F}+\mathrm{M}$ or $\mathrm{G}+\mathrm{M}$ pairs would form via Kozai-Lidov oscillations caused by a tertiary leading the M component on an inclined, eccentric orbit which would then decay due to tidal friction. If this scenario is justified, our observations show that orbital realignment is shorter than orbital circularisation, $a$ contrario with the planet case. All this will soon be published in a series of papers.

\section{Acknowledgements}

This is work conducted under supervision by my PhD surpervisor, Didier Queloz, and in collaboration with a large number of people, notably Andrew Collier Cameron, Don Pollacco, Coel Hellier, Michaël Gillon, David Anderson, Barry Smalley, Nami Mowlavi and Leslie Hebb, to name only a few.

\section{References}

Alibert, Y., Mordasini, C., Benz, W., \& Winisdoerffer, C. 2005, A $\& A$, 434, 343

Bayliss, D. R., Winn, J. N., Mardling, R. A., \& Sackett, P. D. 2010, ApJ, 722, L224

Cameron, A. C., Bouchy, F., Hébrard, G. et al., 2007, MNRAS, 375, 951

Fabrycky, D. \& Tremaine, S. 2007, ApJ, 669, 1298

Gillon, M., Smalley, B., Hebb, L. et al., 2009, A\&A, 496, 259

Giménez, A. 2006, $A \& A$ A 450, 1231

Giménez, A. 2006, ApJ, 650, 408

Goldreich, P. \& Tremaine, S. 1980, ApJ, 241, 425

Gregory, P. C. 2005, in Bayesian Logical Data Analysis for the Physical Sciences

Holt, J. R. 1893, A\& $A$, XII, 646

Hosokawa, Y. 1953, PASJ, 5, 88

Howard, A. W., Marcy, G. W., Bryson, S. T. et al., 2011, ApJ, submitted

Kopal, Z. 1942, ApJ, 96, 399

Lin, D. N. C., Bodenheimer, P., \& Richardon D. C. 1996, Nature, 380, 606

Mandel, K. \& Agol, E. 2002, ApJ, 580, L171

Mayor, M. \& Queloz, D. 1995, Nature, 378, 355

McLaughlin, D. B. 1924, ApJ, 60, 22

Moutou, C., Rodrigo, R. F., Udry, S. et al., 2011, A\&A, submitted

Mowlavi, N., Eggenberger, P., Meynet, G. et al., 2012, A\&A, submitted (arXiv:1201.3628)

Naef, D., Mayor, M., Beuzit, J.-L. et al., 2005, in Proceedings of the 13th Cambridge Workshop on Cool Stars, 833

Nagasawa, M., Ida, S., \& Bessho, T. 2008, ApJ, 678, 498

Pollack, J. B., Hubickyj, O., Bodenheimer, P. et al., 1996, Icarus, 124, 62

Pollacco, D., Skillen, I., Cameron, A. C. et al., 2006, PASP, 118, 1407

Queloz D., Eggenberger, A., Mayor, M. et al., 2000, A\&A, 359, L13

Rasio, F. A. \& Ford, E. 1996, Science, 274, 954

Rossiter, R. A. 1924, ApJ, 60, 15

Sozzetti, A., Torres, G., Charbonneau, D. et al., 2007, ApJ, 664, 1190

Triaud, A. H. M.. J., Queloz, D., Bouchy, F. et al., 2009, A\&A, 506, 377

Triaud, A. H. M.. J., Collier Cameron, A., Queloz, D. et al., 2010, A\&A, 524, 25

Triaud, A.H.M.J., PhD Thesis submitted

Ward, W. R. 1997, Icarus, 126, 261

Winn, J., Fabrycky, D., Albrecht, S. et al., 2010, ApJ, 178, L145 


\section{Discussion}

A. Vidotтo: You mention that $M$ dwarfs transiting/eclipsing in binary systems show $\beta \sim 0$. Are there similar measurements concerning transiting brown dwarfs?

A. TRIAud: Yes. There are four transiting brown dwarfs: CoRoT-3b, CoRoT-15b, WASP30b and LHS-6343c. There are two Rossiter-McLaughlin effect measurements, to my knowledge: CoRoT-3b which is found slightly misaligned but is probably aligned - the data are badly sampled due to the faintness of the target (see Triaud et al. 2009); and WASP-30b, which is found aligned (Triaud et al. in prep).

W. KLEY: Is there any correlation between planetary mass and misalignment angle?

A. Triaud: Yes. At the moment no planet $>5 M_{\mathrm{Jup}}$ is found retrograde (Moutou et al. submitted). Following on the previous comments, the known brown dwarfs and $\mathrm{M}$ dwarfs eclipsing $\mathrm{F}$ and $\mathrm{G}$ primaries are all found aligned at the moment, regardless of the eccentricity, spectral type of the primary or the orbital periods ranging up to 15 days. All those combined point to a correlation with mass.

E. BudDing: Some of the low mass, rapidly rotating cool dwarfs should be affected by spots. Since the Rossiter-McLaughlin effect will be following the motion of the centre of light for the eclipsed component, won't the question of possible misalignment of the rotation axis of the eclipsed star become complicated by the presence of such spots?

A. TRIAUd: Spots can induce two effects: the first is when the planet crosses a spot, then, since the Rossiter-McLaughlin effect is a change in the light centroid, the planet is being hidden and we lose that information. This, if the planet covers exactly a spot, we lose the radial velocity anomaly and recover the Keplerian velocity that is observed in the out-of-transit data. The second case is when spots cover the star inhomogeneously. Let's imagine we have one spot, located in the blue-shifted hemisphere, out of the transit chord. Then, the planet will hide a comparatively larger area on the blue-shifted hemisphere, compared to the red-shifted hemisphere. This would lead to an asymmetry in the RossiterMcLaughlin effect, which could be confused for a misalignment. Such an occurrence may be responsible for the different results obtained independently on WASP-17 (see Triaud et al. 2010 and Bayliss et al. 2010).

E. GUINAN: Is there a relation between orbital alignment and time?

A. Triaud: Yes. This is work which is still unpublished, while being submitted to A\&A. I am reluctant to talk publicly about it, preferring to wait for the referee to give some feedback. To answer the question, I will point out that hot Jupiters found around more massive stars are younger and detectable only for a shorter time (after the star is too big) than those around lower mass stars. Therefore, the observed misaligned systems (found primarily around hot stars) are on average younger systems than the aligned systems which are found around the colder stars. 